\title{
Locational reserve disqualification for distinct scenarios
}

\author{
Joshua D. Lyon, Student Member, IEEE, Muhong Zhang, and Kory W. Hedman, Member, IEEE
}

\begin{abstract}
Reserve requirements promote reliability by ensuring resources are available to re-balance the power system following random disturbances. However, reliability is not guaranteed when reserve dispatch is limited by transmission constraints. In this work, we propose a modified form of reserve requirement that identifies response sets for distinct contingency scenarios. The approach disqualifies reserve from counting towards a particular scenario if transmission constraints are likely to render that reserve undeliverable. A decomposition algorithm for securityconstrained unit commitment dynamically updates the response sets to address changing conditions. Testing on the RTS 96 test case demonstrates the approach applied in tandem with existing reserve policies to avoid situations where reserve is not deliverable due to transmission constraints. Operational implications of the proposed method are discussed.
\end{abstract}

Index Terms-Operating reserve, optimization, power system economics, power system reliability, power system security, reserve requirements, unit commitment.

\section{NOMENCLATURE}

\section{A. Sets}

$G \quad$ Generators and reserve providers; $G(n) \subseteq G$ and $\mathcal{G}(k) \subseteq G$ are the sets at node $n$ and in zone $k$.

$Z \quad$ Zones; $z(c) \in Z$ is the zone of contingency $c$.

$L \quad$ Transmission branches (lines and transformers).

$N \quad$ Nodes; $n(g) \in N$ is the node of resource $g$.

T Time periods.

\section{B. Parameters}

$F_{l} \quad$ Emergency rating of line $l$.

$P T D F_{n l}$ Power transfer distribution factor for line $l$ based on a transfer from $n$ to the reference node.

$\Gamma_{g t}^{c} \quad$ Response set membership; $\Gamma_{g t}^{c}=0$ disqualifies resource $g$ from contingency $c$ in period $t$.

$I_{n} \quad$ Net injection at node $n$ prior to re-dispatch ${ }^{1}$

$R_{g} \quad$ Reserve held by resource $g_{1}^{1}$

$\hat{\Gamma}_{g} \quad$ Response set membership of resource $g$ from the most recent iteration ${ }^{1}$

This work was supported by the NSF under award \#1333646 and by the U.S. Department of Energy for "The Future Grid to Enable Sustainable Energy Systems," an initiative of the Power Systems Engineering Research Center.

Joshua D. Lyon and Muhong Zhang are with the Department of Industrial Engineering and Kory W. Hedman is with the School of Electrical, Computer and Energy Engineering at Arizona State University, Tempe, AZ, 85287 USA (email: joshua.lyon@asu.edu; muhong.zhang@asu.edu; kory.hedman@asu.edu).

\section{Variables}

$f_{l t} \quad$ Power flow on line $l$ in period $t$.

$p_{g t} \quad$ Power produced by generator $g$ in period $t$.

$r_{g t} \quad$ Reserve provided by resource $g$ in period $t$.

$\tilde{r}_{k t}^{x} \quad$ Reserve in zone $k$ classified as deliverable to zone $x$ or contingency $x$ in period $t$.

$S_{k t}^{j} \quad$ Reserve sharing limit from zone $k$ to zone $j$ in period $t$.

$i_{n} \quad$ Net injection at node $n$ after re-dispatch 1

$e_{g} \quad$ Exercised reserve from resource $g_{1}$

$m_{g} \quad$ Undeliverable reserve from resource $g_{1}^{1}$

$\gamma_{g} \quad$ Proportion of reserve that is not deliverable 1

\section{INTRODUCTION}

$\mathbf{T}$ HE N-1 reliability standard requires operators to manage any single contingency without curtailing involuntary loads. After a generator fails, operators have a short time window to restore frequency while satisfying emergency transmission constraints [1]. Dispatchable reserves are the primary recourse for many such contingencies. This paper focuses on scheduling with security-constrained unit commitment (SCUC) in a manner that ensures enough reserve will be deliverable during contingency response. SCUC has a prominent role during day-ahead scheduling because it determines which generators will be committed and clears the day-ahead market (DAM). Physical constraints in SCUC include generator capacity, ramping, and linearized power flows [2], while reserve requirements provide flexibility to help operators satisfy physical requirements when the system state deviates from the forecast.

Reserve requirements specify how much generators must collectively be able to ramp within a prescribed period of time. Like all operating constraints, reserve requirements can force generators to be dispatched out-of-merit. Therefore, it can be expensive to acquire more reserve than is necessary to satisfy reliability targets. The cost of holding reserves may be significant even when market prices are low because prices rarely capture the impact on which generators are committed [3]. Most large balancing authorities use reserve zones to ensure enough reserve is held within import-constrained

\footnotetext{
${ }^{1}$ This term is used when pruning the response set (disqualifying reserve)
} for a particular contingency-period $(c, t)$. 


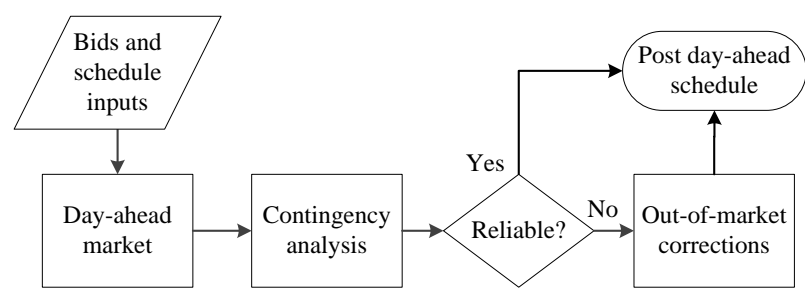

Fig. 1. Sequential day-ahead scheduling process.

regions. However, reserve zones are structurally limited and are expected to address a wide range of uncertainty: requirements are inevitably overly conservative for some scenarios and insufficient for others.

Reserve requirements are enforced through SCUC so that the solution satisfies reliability targets. SCUC clears the DAM to establish an initial schedule that facilitates market settlements. This marks the beginning of the sequential scheduling process described in Fig. 1. After the market has been cleared, operators evaluate reliability and apply out-of-market corrections (OMCs) whenever the market solution is unreliable [4] (these interventions are classified as exceptional dispatches in CAISO or out-of-merit energy/capacity in ERCOT [5], [6]). Reserve requirements are generally not enforced during contingency analysis because corrective actions may involve any reserves that are physically available. If some reserves are found to be undeliverable, MISO and ISO-NE manually disqualify them so that requirements must be met by reserves at other locations [7]-[9]. Such OMCs are not co-optimized with other scheduling decisions and are not reflected in day-ahead prices. Therefore, it is worthwhile to seek improvements to the DAM model so that fewer OMCs are necessary.

Stochastic programs reduce the need for OMCs by modeling the forecasted state and recourse decisions for a set of scenarios. Several papers on stochastic unit commitment incorporate recourse transmission constraints [10]-[16], thereby implicitly determining optimal reserve locations. This precision is computationally expensive, however, making full implementation intractable for large systems. Robust optimization can be less computationally burdensome [17], [18] but is still much slower than deterministic models [19]. Operators will continue to rely on reserve requirements until these computational challenges can be overcome. Even as computational advances are achieved, the use of proxy reserve requirements will continue because they provide an intuitive way to satisfy necessary conditions for reliability.

This paper proposes a new reserve requirement based on response sets. A response set is a group of entities eligible to provide reserve for a particular scenario. Scenario-specific response sets help SCUC anticipate what corrective actions will be feasible for different contingencies in different periods. This work focuses on generator contingencies, but the structure is theoretically compatible with any other type of uncertainty. We propose a decomposition algorithm that updates response sets while solving SCUC. The algorithm mimics Benders' decomposition for stochastic programs but is less computationally intensive and relates to existing reserve disqualification practices. Removing a resource from a response set is equivalent to disqualifying reserve for a single contingency; therefore, the methodology can be characterized as a generalized reserve disqualification procedure. The algorithm is passive in the sense that it only overrides traditional reserve requirements when reserve deliverability assumptions are violated. The proposed approach may be integrated with the DAM or used as an OMC strategy to improve reliability in an economical manner.

The remainder of this paper is organized as follows: Section II describes a baseline zonal approach for reserve requirements in SCUC. Section IIII describes the limitations of zonal reserve requirements. Section IV describes the form and implementation of the proposed method. Section $\mathrm{V}$ presents numerical results. Finally, Section $\mathrm{VI}$ concludes the paper and summarizes potential future work.

\section{BASELINE RESERVE REQUIREMENTS}

Stochastic formulations include recourse decisions that describe how the operator may respond for a set of contingencies. Incorporating a full network model for all contingencies quickly becomes intractable for large systems due to how the problem size increases. Zones are used in practice to approximate transmission constraints and enable deterministic formulations. References [20]-[23] provide means to determine reserve zones. It is well-known that zones do not perfectly characterize network flows because they estimate cross-border flows and ignore intra-zonal congestion [24]. Nonetheless, zones are ubiquitous for reserve requirements because they allow operators to model transmission constraints on recourse decisions in an intuitive and computationally efficient way.

The baseline reserve model used in this work is inspired by the market model of ISO-NE [25]. Two small adjustments are made to the formulation. The first (and most trivial) difference is that the model only considers 10-minute reserves, which are procured for $\mathrm{N}-1$ contingency response. The second change is a departure from nested zones, which suit the ISO-NE system but may be inappropriate for general networks.

The reserve model proposed by [25] introduces variables that describe how much reserve can be shared between zones. A minimalistic representation is shown by (1)-(3). The variable $\tilde{r}_{k t}^{j}$ represents how much reserve in zone $k$ is classified as deliverable to zone $j$ in period $t$. Equation (1) requires there to be enough portable reserve to cover the loss of any generator, (2) models reserve held within the zones, and (3) limits how much reserve may be shared between zones.

$$
\begin{array}{lr}
\sum_{k \in Z} \tilde{r}_{k t}^{j} \geq p_{c t}+r_{c t} & \forall j \in Z, c \in \mathcal{G}(j), t \in T \\
\tilde{r}_{k t}^{j} \leq \sum_{g \in \mathcal{G}(k)} r_{g t} & \forall j \in Z, k \in Z, t \in T \\
\tilde{r}_{k t}^{j} \leq S_{k t}^{j} & \forall j \in Z, k \in Z, t \in T
\end{array}
$$

The above formulation is appropriate for modeling reserve sharing between adjacent zones. The sharing bounds $(S)$ may be based on off-line analysis. For example, ISO-NE dynamically updates $S_{k t}^{j}$ to be the (predefined) transfer capability from zone $k$ to $j$ less the interface flows in the modeled system state [25]. Reference [25] differs from the above formulation 


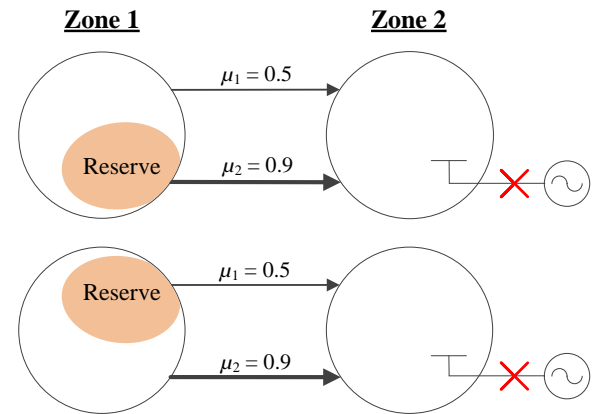

Fig. 2. Transfer capability depends on reserve locations relative to highly utilized $(\mu)$ lines.

because it models sharing across multiple layers of nested zones; ISO-NE only constrains reserve sharing from parent to child zones, which is reasonable when power consistently flows in the same direction. The work in this paper is applicable for both (1)-(3) and nested zones alike.

\section{LIMITATIONS OF TRADITIONAL RESERVE REQUIREMENTS}

Traditional reserve requirements suffer from two inherent limitations. First, reserve sharing estimates may be imprecise because they are based on off-line studies performed before the system state is known. These studies must anticipate the injection and withdrawal locations. Reserve locations are important because Kirchhoff's laws map power flows to the locational injections and more power can be transferred between points that have similar shift factors on critical lines. Consider the example in Fig. 2. Although the system states are indistinguishable at the zonal level, more reserve can be transferred to zone two in the second case because reserve is held at locations that prefer the path that has more residual capacity. Zonal models are inherently imprecise because they assume that all members of the same zone have equivalent shift factors on congested interfaces.

Ideally, reserve sharing would reflect contingency-specific transfer capabilities. MISO acknowledges this concern and uses simulations to estimate reserve sharing capabilities on a per-contingency basis [20]. MISO fixes the minimum reserve quantity for each zone according to the contingency with the largest loss minus import capability. The largest contingency is not necessarily the most severe because other contingencies may hinder reserve imports by reducing counter-flows on critical lines. Although MISO's requirements are contingencyspecific, they are derived prior to SCUC and may be suboptimal whenever the schedule conflicts with the projected system state.

The second limitation is that traditional reserve requirements assume that no reserve deliverability issues will be caused by local congestion within a zone. One way to mitigate intra-zonal congestion is to procure a reserve margin for more dispatching freedom to alleviate congestion [26]. For example, PJM requires reserve to exceed roughly 1.5 times the largest contingency and WECC requires reserve to cover $6 \%$ of load plus $3 \%$ of exports [27], [28]. These margins can protect against general uncertainties beyond $\mathrm{N}-1$, but they are not an ideal way to improve reserve deliverability because operators lack means to control reserve locations within the zones.

\section{LOCATIONAL DISQUALIFICATION}

The limitations of traditional reserve requirements stem from the fact that they do not control the location of reserves within zones. To address this issue, we use contingency-specific response sets that suspend reserves held at unfavorable locations. The new reserve model is described by (4)-(6). The zonal reserve variable $\tilde{r}_{k t}^{c}$ is now indexed by contingency $c$ (instead of zone $j$ ) and represents reserve in zone $k$ that is classified as deliverable for contingency $c$. The parameter $\Gamma_{g t}^{c} \in[0,1]$ limits the proportion of reserve that is classified as deliverable. $\Gamma$ has no authority over actual contingency response, but can anticipate what reserves will be deliverable so that SCUC provides a more reliable solution.

$$
\begin{array}{lr}
\sum_{k \in Z} \tilde{r}_{k t}^{c}=p_{c t}+r_{c t} & \forall c \in G, t \in T, \\
\tilde{r}_{k t}^{c} \leq \sum_{g \in \mathcal{G}(k)} \Gamma_{g t}^{c} r_{g t} & \forall c \in G, k \in Z, t \in T, \\
\tilde{r}_{k t}^{c} \leq S_{k t}^{z(c)} & \forall c \in G, k \in Z, t \in T
\end{array}
$$

Each response set must hold enough reserve to replace the underlying generator $c$, subject to the same reserve sharing constraints as the baseline model: $S_{k t}^{z(c)}$ limits sharing from zone $k$ to the contingency zone $z(c)$. The new reserve requirements (4)-(6) are equivalent to (1)-(3) when all $\Gamma=1$. Setting $\Gamma_{g t}^{c}=0$ disqualifies the reserve provided by resource $g$. The proposed formulation is more general than MISO and ISO-NE practices because reserve disqualification is indexed by contingency and $\Gamma$ may take any value between zero and one. Equations (4)-(6) enable contingency-specific management of reserve sharing and intra-zonal congestion, provided that proper values for $\Gamma$ can be determined.

Fig. 3 outlines a two-stage decomposition algorithm that iteratively updates $\Gamma$ while solving SCUC. First, SCUC is solved using relaxed response sets (hereon assume that all $\Gamma_{g t}^{c}=1$ to begin) to obtain a solution that is economical but not necessarily reliable. Contingency analysis then identifies contingencies that have insufficient deliverable reserves. The response sets are then pruned (i.e., made more restrictive by reducing $\Gamma$ ) for these contingencies and the restricted reserve requirements are passed back to SCUC. Each update can be viewed as a reliability cut because it restricts the amount of reserve designated as deliverable. These updates are analogous to the feasibility cuts generated by Benders' decomposition for two-stage stochastic programs. However, pruning $\Gamma$ may remove larger portions of the feasible space than Benders' cuts: the proposed algorithm does not guarantee optimality but fewer iterations may be needed to converge to a reliable solution.

The proposed reserve disqualification procedure in Fig. 3 may be applied within the DAM or as a mechanism to determine OMCs. It is preferable to determine a reliable solution within DAM procedures, but this frequently does not occur due to time limitations. When the proposed DAM solution is not reliable, market operators adjust the market solution using OMCs. For 
(updated reserve requirements)

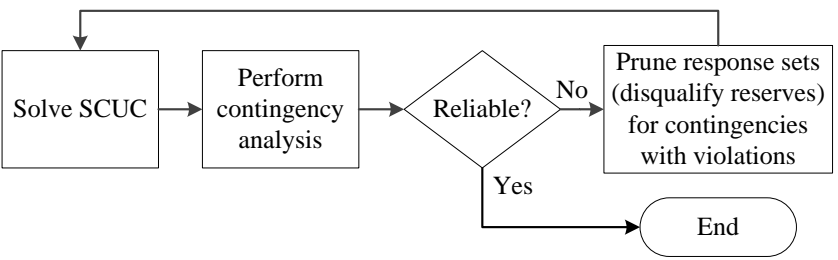

Fig. 3. Decomposition algorithm for SCUC with reserve disqualification.

market purposes, these OMCs are often not allowed to decommit units that were committed in the DAM framework [5]. Such restrictions are uneconomical because they create a separation between DAM and OMC decisions.

Reserve settlement schemes may be devised to distinguish resources at favorable locations. For example, payments may be based on the service provided to individual contingencies based on the shadow prices (dual variables) of (5). Such a scheme could capture more of a locational aspect than traditional markets where the reserve price is the same for all resources within a zone. Improved reserve policies provide an opportunity to redesign settlements to better reflect the quality of service provided by individual resources. Analysis of market design deserves thorough attention and is deferred to future work. The focus of this work is on how reserve disqualification affects operational costs and reliability.

\section{A. Pruning the response set for contingency-period $(c, t)$}

This section proposes a mathematical program to prune the response set for a single unreliable contingency in a particular period. The outcome of this model is additional reserve disqualifications that are passed back to SCUC in the process described by Fig. 3. A functional pruning algorithm should be fast, fair, and effective. To be sufficiently fast, it should solve quicker than SCUC. To be fair, it should not unduly disqualify resources that have deliverable reserve and thereby exclude them from remuneration. To be effective, the pruning algorithm should return a $\Gamma$ that improves the reliability of the SCUC solution in an economical manner. This section outlines the formulation and Section $\nabla$ evaluates the economical effectiveness of the proposed approach.

We propose a linear program to prune response sets after an initial SCUC solution is available. The model considers spinning reserves but can be generalized to include nonspinning reserves. For a particular contingency, let $I$ represent the net injections (generation minus load) from the incumbent SCUC solution prior to re-dispatch, $R$ be the available reserves, and $\hat{\Gamma}$ be the ruling disqualification factors from the previous iteration. Equations (7)-(10) measure the aptitude of the existing response set; the response set is sufficient if there is a solution to the linear power flow equations $(7)-(10)$. Equations (7) and (8) are flow balance and transmission constraints, $(9)$ models locational injections, and (10) constrains reserve availability. More information on linearized power flows, including a description of PTDFs, can be found in [29].

$$
\begin{array}{ll}
\sum_{n \in N} i_{n}=0, & \\
-F_{l} \leq \sum_{n \in N} P T D F_{n l} i_{n} \leq F_{l}, & \forall l \in L, \\
i_{n}=I_{n}+\sum_{g \in G(n)} e_{g}, & \forall n \in N, \\
0 \leq e_{g} \leq R_{g} \hat{\Gamma}_{g}, & \forall g \in G
\end{array}
$$

Equations (7)-(10) may not be satisfied if congestion prevents reserves from being delivered. The pruning model $\left(P_{t}^{c}\right)$ defined below relaxes the power flow and is guaranteed to be feasible whenever the reserve quantity exceeds the size of the contingency. Constraints $(14)-(18)$ separate corrective actions into two components: reserve dispatched through $e$ is injected as normal and reserve dispatched through $m$ is moved directly to the location of the contingency $n(c)$ without using any transmission capacity. A trivially feasible solution is to dispatch all reserve using $m$ so that the nodal injections are the same as in the pre-contingency state. The variable $\gamma$ identifies resources with non-deliverable reserve and the objective (11) minimizes the amount of reserve marked as non-deliverable.

$$
\begin{array}{ll}
\operatorname{minimize} & \sum_{g \in G} R_{g} \gamma_{g}, \\
\sum_{n \in N} i_{n}=0, & \\
-F_{l} \leq \sum_{n \in N} P T D F_{n l} i_{n} \leq F_{l}, \quad & \forall l \in L, \\
i_{n}=I_{n}+\sum_{g \in G(n)} e_{g}, \quad \forall n \in N \backslash n(c), \\
i_{n}=I_{n}+\sum_{g \in G(n)} e_{g}+\sum_{g \in G} m_{g}, \quad n=n(c), \\
0 \leq e_{g} \leq R_{g} \hat{\Gamma}_{g}\left(1-\gamma_{g}\right), & \forall g \in G, \\
0 \leq m_{g} \leq R_{g} \hat{\Gamma}_{g} \gamma_{g}, & \forall g \in G, \\
0 \leq \gamma_{g} \leq 1, & \forall g \in G
\end{array}
$$

The respective response set is updated by $\Gamma_{g t}^{c}=\hat{\Gamma}_{g}\left(1-\gamma_{g}^{*}\right)$, where $\gamma_{g}^{*}$ is from the optimal solution to $\left(P_{t}^{c}\right)$. All resources with $\gamma_{g}^{*}=1$ are removed from the response set because their reserve was not deliverable during simulation of a coordinated re-dispatch. The pruned reserves may not count towards the reserve requirement (5) in SCUC during the next iteration. This approach is fair because it avoids undue disqualification by minimizing the amount of pruning at each iteration. However, the updated response sets may become invalid if subsequent SCUC solutions are too dissimilar from earlier solutions.

The pruning algorithm need not be convex. For example, problem $\left(P_{t}^{c}\right)$ can be updated to include non-spinning reserves. Substituting an AC power flow would provide a mechanism to disqualify reserves based on voltage violations. There has recently been greater interest in using topology control to alleviate congestion [30], and $\left(P_{t}^{c}\right)$ may be amended to reflect such corrective actions. The proposed algorithm can generally consider a wider scope of decisions than Benders decomposition 


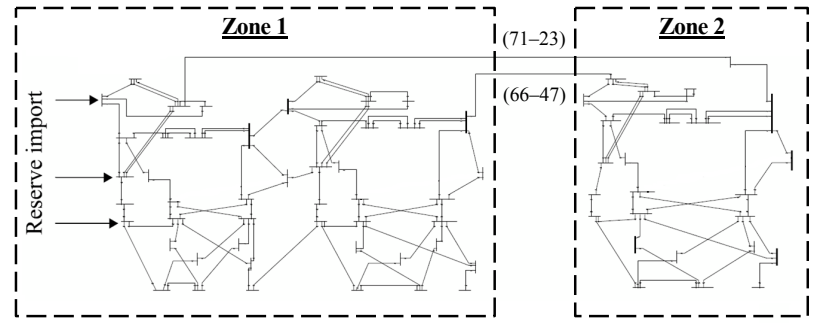

Fig. 4. Reserve zones for the modified IEEE 73-bus test case with up to 200 MW of reserve imports.

because the corrective actions do not need to be convex [31].

Computationally, the pruning algorithm is comparable to contingency analysis that operators solve on a frequent basis. Large-scale problem instances are tractable because response sets can be pruned in parallel for different contingencies.

Response sets may be applied to other reserve requirements that are not covered in this paper. If a different reserve model is used, then the bounds on $e$ (16) and $m$ (17) should be adjusted to reflect the reserve up and reserve down requirements enforced in SCUC and the relaxation of (7)-(10) should be amended so that $m$ corresponds to the location of the disturbance.

\section{ANALYSIS AND RESUlts: RTS 96 TEST CASE}

The proposed reserve disqualification procedure is evaluated on seven days across the peak week of the IEEE 73-bus (RTS 96) test case [32], [33]. Weekend testing captures the impact of the proposed method on off-peak days. Modifications to the test case follow [26]: line (11-13) is removed; $480 \mathrm{MW}$ of load is shifted from buses $14,15,19$, and 20 to bus 13 ; and the capacity of line (14-16) is decreased to $350 \mathrm{MW}$. These modifications affect each of the three identical areas within the system. A small amount of congestion is induced by tripling the capacity of inexpensive hydro power in the area consisting of buses 1-24 and removing hydro from all other areas. The test case is treated as a region within a larger system that can import up to $200 \mathrm{MW}$ of reserve evenly across buses 3, 15, and 17. This paper analyzes the use of 10-minute spinning reserves to respond generator contingencies.

We identify reserve zones using the partitioning method of [22], which uses statistical clustering so that reserve can compensate for disturbances in the same zone with little change to flow on critical lines. We identify critical lines by solving SCUC for the peak day using a single zone and then performing contingency analysis. Slack variables allow transmission violations when insufficient reserves are deliverable. Line (73-21) is classified as the only critical line because no other line limit is ever relaxed to improve access to reserves. Fig. 4 shows the resulting zone partition. Zone one comprises nodes (1)-(48) and zone two comprises nodes (49)-(73). Intra-zonal congestion is rarely seen in either zone but interface line (73-21) often limits reserve imports into zone two. This analysis will demonstrate that reserve disqualification can still be effective when only one line is congested.

All testing is performed using CPLEX v12.5 on an 8-core 3.6GHz computer with $48 \mathrm{~GB}$ of memory. Unless otherwise
TABLE I

AVERAGE BASELINE RESUlTS OVER THE 7 TEST DAYS.

\begin{tabular}{ccccccc}
\hline $\begin{array}{c}\text { Policy } \\
(\alpha)\end{array}$ & Cost & $\begin{array}{c}\text { Time } \\
(\mathrm{mins})\end{array}$ & $\begin{array}{c}\text { Optimality } \\
\text { gap }\end{array}$ & $\begin{array}{c}\text { E[viol] } \\
(\mathrm{MW})\end{array}$ & \# viol & $\begin{array}{c}\max \text { viol } \\
(\mathrm{MW})\end{array}$ \\
\hline $45 \%$ & $\$ 2,393,971$ & 4.8 & $0.17 \%$ & 0.004 & 0.9 & 1.9 \\
$50 \%$ & $\$ 2,382,857$ & 4.8 & $0.16 \%$ & 0.032 & 2.9 & 5.8 \\
$55 \%$ & $\$ 2,374,080$ & 3.8 & $0.13 \%$ & 0.046 & 5.1 & 10.2 \\
$60 \%$ & $\$ 2,370,421$ & 3.9 & $0.12 \%$ & 0.208 & 12.9 & 28.2 \\
$65 \%$ & $\$ 2,369,969$ & 3.1 & $0.11 \%$ & 0.385 & 17.3 & 42.8 \\
\hline stochastic & $\$ 2,374,328$ & 60.2 & $0.17 \%$ & 0 & 0 & 0 \\
\hline
\end{tabular}

stated, SCUC is terminated after five minutes or upon reaching an optimality gap of $0.1 \%$. The model includes 99 generators and 24 hours, which translates to 2376 response sets.

\section{A. Baseline reserve requirements}

The baseline reserve requirements (1)-(3) oblige the user to specify reserve sharing limits. We test several different policies and compare them post hoc. Although operators do not have time to compare many alternatives in the midst of making day-to-day decisions, this strategy allows us to evaluate the proposed approach alongside both conservative and liberal reserve sharing policies.

Constraints (19)- 21$]$ are introduced to limit reserve sharing between zones. The sharing limit between each zone and itself is an arbitrarily large value $M$, which should exceed reserves to avoid imposing an artificial limit on reserve availability. The emergency capacities for the zonal interface, which comprises lines (73-21) and (66-47), sum to $1250 \mathrm{MW}$. We define the sharing limit between the adjacent zones in relation to the import headroom on these lines. The parameter $\alpha \in[0,1]$ de-rates the sharing capability because the interface lines may not reach their limits simultaneously.

$$
\begin{array}{llrl}
S_{1 t}^{1} & =S_{2 t}^{2}=M, & & \forall t \in T, \\
S_{1 t}^{2} & =\alpha 1250-f_{(73-21), t}-f_{(66-47), t}, & & \forall t \in T, \\
S_{2 t}^{1} & =\alpha 1250+f_{(73-21), t}+f_{(66-47), t}, & & \forall t \in T
\end{array}
$$

Five reserve sharing policies are tested corresponding to $\alpha=\{0.45,0.50,0.55,0.60,0.65\}$. Table I] summarizes the average results over the seven test days. Conservative reserve sharing policies (small $\alpha$ ) result in higher operating costs but tend to be more reliable. Three statistics measure reliability: "E[viol]" is the expected sum of transmission violations from contingency analysis, where contingency probabilities are inferred from the mean times to failure [33]; "\# viol" is the number of contingencies with some violation; and "max viol" is the maximum sum of transmission violations for any contingency. Sections $\mathrm{V}-\mathrm{B}$ and $\mathrm{V}-\mathrm{C}$ evaluate the use of reserve disqualification to eliminate violations when the initial solution is not reliable.

Table [ also summarizes results from the deterministic equivalent to a stochastic program, which was solved to obtain proper optimality gaps for the proposed algorithms. This extensive form SCUC explicitly models corrective actions for a limited set of contingencies. A single reserve zone requires reserve to exceed the largest contingency and corrective actions 
are explicitly modeled to ensure reserve deliverability. The stochastic program identifies a solution that is about $0.8 \%$ cheaper than the most reliable baseline policy.

Note that solution times for stochastic SCUC are prohibitively long and the extensive form model does not scale well for large systems. The reported solution time of 60 minutes in Table I] was only obtained by limiting contingencies to those that failed contingency analysis at some point during the proposed decomposition algorithm. Without such prior information, the solution times are generally much longer.

\section{B. Reserve disqualification as an out-of-market correction}

Reserve disqualification is evaluated as an ex-post mechanism to improve the reliability of the DAM solution. Such procedures have been classified in this paper as out-of-market corrections (OMCs). To be consistent with market rules, all generators that are committed by the DAM model must stay committed. Therefore, this particular application of the algorithm from Fig. 3 will not de-commit any units that were committed by the market model.

Two different reserve disqualification strategies are considered. The first approach applies the proposed algorithm and is referred to as generalized reserve disqualification (GRD). The second approach disqualifies reserves uniformly across contingencies and is referred to as traditional reserve disqualification (TRD). The pruning algorithm from Section IV-A is adjusted to accommodate TRD. The first adjustment discharges the idea of partial disqualification by adding an integrality constraint on $\gamma$. The response sets are then pruned one contingency at a time and global disqualification is enforced by specifying that resources disqualified from one contingency may not offer reserves for any other contingency during the same period. These amendments provide an automated approach that mimics the manual reserve disqualifications used by operators today.

Fig. 5 illustrates the progress of the reserve disqualification procedures on the highest and lowest load days (Tuesday and Sunday) of the peak week. SCUC determines the operating costs (x-axis) and contingency analysis measures the reliability (y-axis). The red diamonds represent DAM solutions with varying reserve sharing policies $(\alpha)$ : small values of $\alpha$ tend to be more reliable but are also more expensive because they require additional reserve within local areas. The GRD and TRD algorithms start with these initial solutions, disqualify reserves, and solve SCUC again with reserves procured from different locations. The dotted lines represent the progress at each iteration. Both procedures converge to $\mathrm{N}-1$ reliable solutions that are economical compared to the most reliable DAM solution. This suggests that reserve disqualification adds robustness to the baseline policy because operators can recover from unreliable solutions and still maintain reasonable operating costs. Fig. 5 also demonstrates that GRD consistently outperforms TRD, suggesting that it is beneficial to define different response sets for distinct contingencies.

At first glance, it may seem counterintuitive that an offpeak day would require a more conservative reserve sharing policy to achieve the same level of reliability. The reason
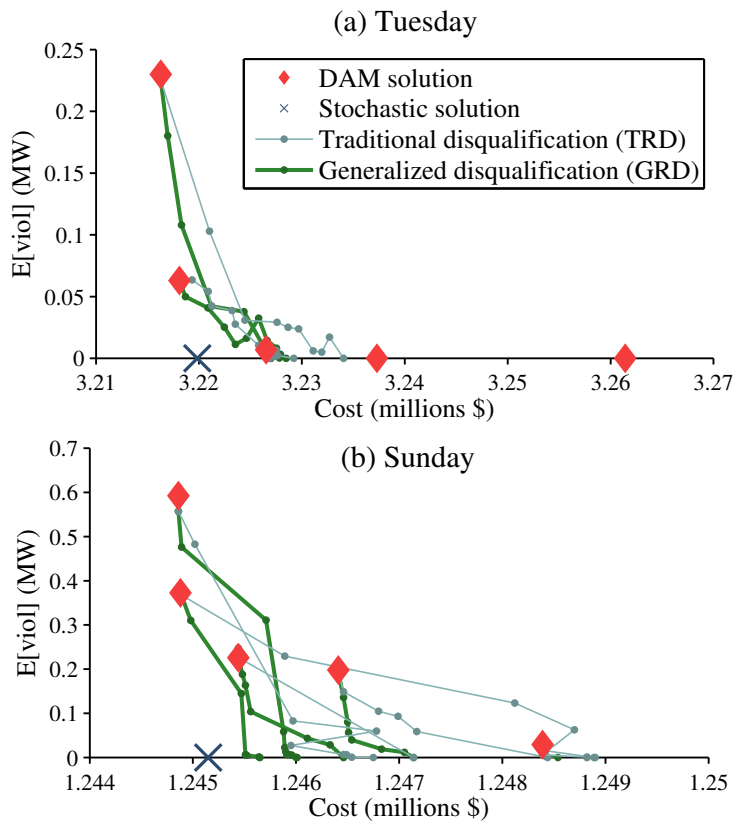

Fig. 5. Progress of the iterative reserve disqualification algorithm when applied to unreliable DAM solutions.

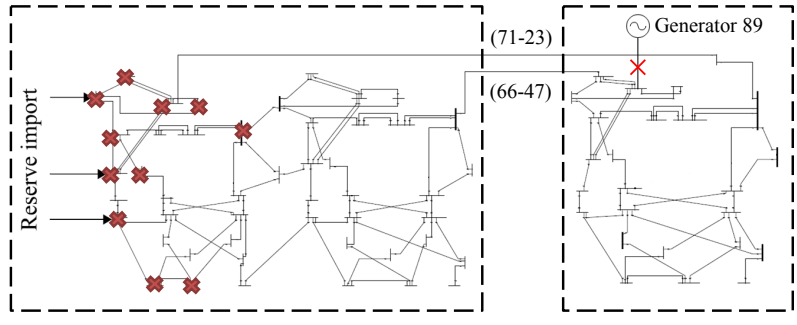

Fig. 6. Locations with reserves disqualified from the response set for generator 89 on hour two of Sunday, day 357. (Line (73-21) is heavily utilized).

for this phenomenon is that generation serving the base load creates relatively little flow on interface line (66-47). When a contingency occurs in zone two, the interface residual capacity is deceptively large because reserve imports are limited by the bottleneck on line (73-21) while line (66-47) remains relatively underutilized. The accuracy of reserve sharing models is not driven by the amount of congestion but how well the reserve sharing model anticipates the influence of congestion on corrective actions. The underlying issue is the same as in Fig 2. In this case, reserve disqualification is effective on low load days because it encourages reserve to be held at locations that favor underutilized paths. Fig. 6 shows that reserves proximal to the heavily utilized line (73-21) are disqualified from the response set for a large contingency in zone two.

Fig. 7f and Fig. 7p describe the final costs (after OMCs have been applied) relative to the stochastic solution. The solutions derived using GRD are more consistently close to the lower bound obtained from the stochastic model. Conservative reserve sharing policies $(\alpha=0.45$ and 0.50 ) often underperform because SCUC obtains an overly conservative solution at the expense of economics. These results suggest that it may be more economical to acquire a risky initial solution (large $\alpha$ ) and then improve reliability through OMCs instead of starting off with an overly conservative reserve sharing policy. 
(a)

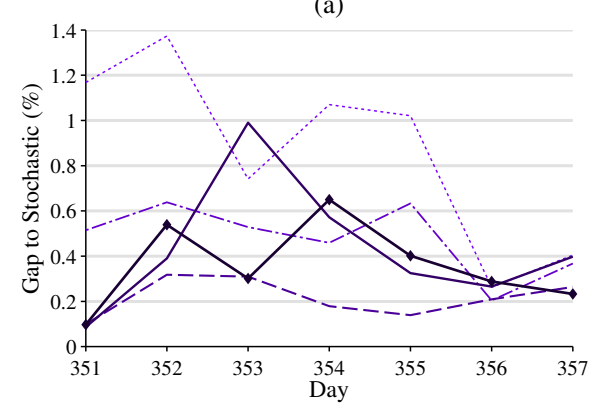

(b)

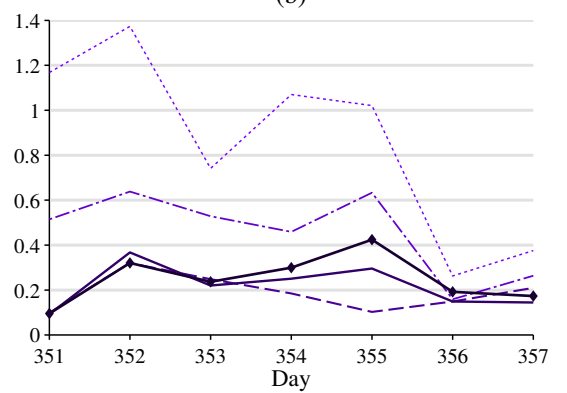

(c)

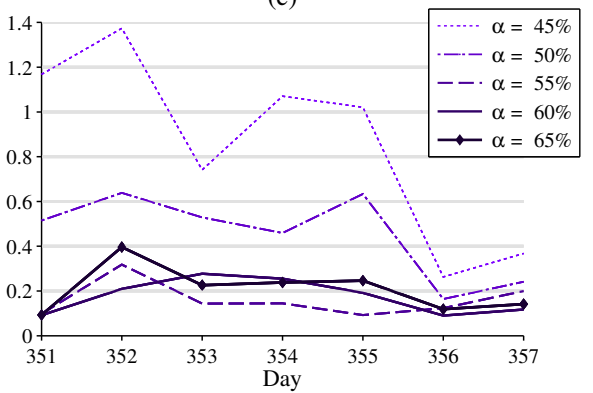

Fig. 7. Final costs for N-1 reliable solutions relative to stochastic programming. (a) OMCs using traditional reserve disqualification. (b) OMCs using generalized reserve disqualification. (c) DAM using generalized reserve disqualification.

TABLE II

COMPUTING STATISTICS FOR THE 7 TEST DAYS.

\begin{tabular}{cccccccc}
\hline \multirow{2}{*}{$\begin{array}{c}\text { Policy } \\
(\alpha)\end{array}$} & \multicolumn{3}{c}{$\#$ iterations } & & \multicolumn{3}{c}{ Time (min) } \\
\cline { 2 - 4 } \cline { 7 - 8 } \cline { 6 - 8 } & Min & Median & Max & & Min & Median & Max \\
\hline $45 \%$ & 1 & 1 & 8 & & 2.6 & 5.2 & 33.9 \\
$50 \%$ & 1 & 1 & 10 & & 5.1 & 5.2 & 37.0 \\
$55 \%$ & 1 & 7 & 20 & & 1.1 & 32.5 & 104.7 \\
$60 \%$ & 6 & 11 & 18 & & 21.0 & 41.5 & 71.2 \\
$65 \%$ & 5 & 11 & 18 & & 15.1 & 33.7 & 97.2 \\
\hline
\end{tabular}

\section{Reserve disqualification for the day-ahead market}

In Section V-B, reserve disqualification was applied after the market model terminated. In this section, the proposed algorithm is tested as a means to clear the DAM itself. Fig. 7k shows that the final costs are consistently lower when reserve disqualification is applied within the DAM, which can be attributed to there being no restrictions on generator commitments across iterations.

Table [II summarizes the total time and the number of iterations to converge to a reliable solution. The majority of time is spent solving SCUC and relatively little effort is spent on contingency analysis and pruning response sets. The proposed algorithm can take 20 iterations to eradicate all violations. Such worst-case convergence times may be unacceptable for some operators. Fortunately, the first iterations tend to be the most influential because pruning is more aggressive when violations are abundant. Fig. 8 plots E[viol] against the improvement to reliability at each iteration. The biggest reliability gains are obtained in early iterations, followed by a long tail of small changes as E[viol] approaches zero. Fig. 8 shows that the majority of iterations occur after the incumbent solution is already nearly reliable.

\section{Practical considerations}

Many ISOs already use iterative approaches to clear the DAM, and it may be practical to incorporate response set pruning with minimal change to existing procedures. The number of iterations is a key factor in consideration because time is typically limited when clearing the DAM. The results in Table II show that a large number of iterations may be necessary before a reliable solution is reached.

Several options are available when time is scarce. First, operators may adopt conservative baseline reserve requirements

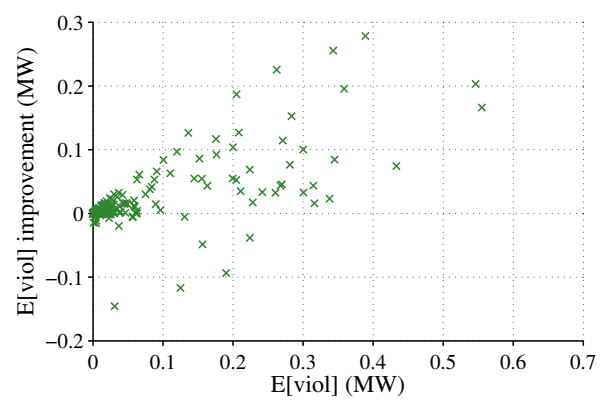

Fig. 8. Reliability vs. reliability improvement at each iteration.

to encourage the initial solution to be more reliable. Second, operators may use off-line analysis to identify initial response sets that require fewer updates. Finally, OMCs may still be applied when a reliable solution is not found within the DAM time limit. The proper balance between these strategies depends on the time restrictions, the average performance of the baseline model, and the operator's tolerance for relying on OMCs.

The proposed approach can improve identification of scarce resources. There is potential for new market settlement schemes to compensate reserve providers on a more locational basis as compared to the zonal ancillary service markets that exist today. For example, the disqualified resources shown in Fig. 6 provide a low quality of service for that particular contingency and need not receive the same zonal price as resources at more favorable locations. Development of such market settlement policies is left to future work.

\section{CONClusions}

Transmission constraints can make reserves unavailable in ways that are hard to anticipate before the SCUC solution is available. As a result, operators disqualify reserves that fall behind unanticipated transmission bottlenecks. Such outof-market changes can be expensive because they are not co-optimized with the DAM decisions.

The proposed reserve disqualification procedure can be used as a heuristic for the DAM or as an improved OMC mechanism. Reserves are disqualified on a per-contingency basis to address the distinct congestion patterns that may arise following different contingencies in different periods. The decomposition algorithm solves SCUC and bases reserve disqualification on system conditions from the incumbent solution. The model 
is able to better characterize scarce resources and thereby improve economics and promote better prices. This algorithmic approach also improves upon traditional OMCs where operators manually disqualify reserves. Testing on the RTS 96 test case demonstrates reduced costs to protect against generator contingencies, particularly when the reserve locations within zones have dissimilar effects on critical lines.

With the upcoming advent of intermittent resources, transmission bottlenecks will become harder to predict and traditional models will be less justifiable because the projected costs under forecasted conditions may poorly reflect the realized costs. New optimization tools and algorithms are needed to determine cost effective solutions within practical time frames for large-scale systems. Future work should extend the proposed approach to help mitigate uncertainty due to intermittent resources as well as create a generalized reserve disqualification procedure for transmission contingencies. Future work is also needed for new market designs and settlement policies for ancillary services markets that would employ such reserve response set rules.

\section{REFERENCES}

[1] NERC, Standard BAL-002-O - Disturbance Control Performance, Feb 2014. [Online]. Available: http://www.nerc.com/pa/Stand/Reliability\% 20Standards\%20Complete\%20Set/RSCompleteSet.pdf

[2] H. Pandžić, T. Qiu, and D. S. Kirschen, "Comparison of state-of-the-art transmission constrained unit commitment formulations," in IEEE PES General Meeting, Jul. 2013.

[3] R. P. O’Neill, P. M. Sotkiewicz, B. F. Hobbs, M. H. Rothkopf, and W. R. Stewart Jr., "Efficient market-clearing prices in markets with nonconvexities," Eur. Jour. of Oper. Res., vol. 164, no. 1, pp. 269-285, Jul. 2005.

[4] Y. Al-Abdullah, M. A. Khorsand, and K. W. Hedman, "Analyzing the impacts of out-of-market corrections," in IREP Symp., Aug. 2013.

[5] CAISO, "2012 annual report on market issues \& performance," Apr. 2013. [Online]. Available: http://www.caiso.com/Documents/ 2012AnnualReport-MarketIssue-Performance.pdf

[6] ERCOT, "Report on existing and potential electric system constraints and needs," Dec. 2007.

[7] FERC, "Docket no. ER11-2794-000 - order conditionally accepting tariff revisions - MISO," Jun. 2011. [Online]. Available: http://www ferc.gov/EventCalendar/Files/20110628160939-ER11-2794-000.pdf

[8] ISO-NE, SOP-RTMKTS.0060.0020 Monitor System Security v57, Feb. 2013. [Online]. Available: http://www.iso-ne.com/rules_proceds/ operating/sysop/rt_mkts/sop_rtmkts_0060_0020.pdf

[9] ISO-NE, SOP-RTMKTS.0120.0030 Implement Transmission Remedial Action v20, Jun. 2012. [Online]. Available: http://www.iso-ne.com/rules_ proceds/operating/sysop/rt_mkts/sop_rtmkts_0120_0030.pdf

[10] J. M. Arroyo and F. D. Galiana, "Energy and reserve pricing in security and network-constrained electricity markets," IEEE Trans. Power Syst. vol. 20, no. 2, pp. 634-643, May 2005.

[11] F. D. Galiana, F. Bouffard, J. M. Arroyo, and J. F. Restrepo, "Scheduling and pricing of coupled energy and primary, secondary, and tertiary reserves," Proc. IEEE, vol. 93, no. 11, pp. 1970-1983, Nov. 2005.

[12] F. Bouffard, F. D. Galiana, and A. J. Conejo, "Market-clearing with stochastic security - part I: Formulation," IEEE Trans. Power Syst., vol. 20, no. 4, pp. 1818-1826, Nov. 2005.

[13] L. Wu, M. Shahidehpour, and T. Li, "Stochastic security-constrained unit commitment," IEEE Trans. Power Syst., vol. 22, no. 2, pp. 800-811, May 2007.

[14] L. Wu, M. Shahidehpour, and T. Li, "Cost of reliability analysis based on stochastic unit commitment," IEEE Trans. Power Syst., vol. 23, no. 3 , pp. 1364-1374, Aug. 2008.

[15] K. W. Hedman, M. C. Ferris, R. P. O'Neill, E. B. Fisher, and S. S Oren, "Co-optimization of generation unit commitment and transmission switching with N-1 reliability," IEEE Trans. Power Syst., vol. 25, no. 2 , pp. 1052-1063, May 2010.

[16] H. A. Hejazi, H. R. Mohabati, S. H. Hosseinian, and M. Abedi, "Differential evolution algorithm for security-constrained energy and reserve optimization considering credible contingencies," IEEE Trans. Power Syst., vol. 26, no. 3, pp. 1145-1155, Aug. 2011.
[17] D. Bertsimas, D. B. Brown, and C. Caramanis, "Theory and applications of robust optimization," Siam Rev., vol. 53, no. 3, pp. 464-501, Aug. 2011.

[18] A. Street, F. Oliveira, and J. M. Arroyo, "Contingency-constrained unit commitment with N-k security criterion: A robust optimization approach," IEEE Trans. Power Syst., vol. 26, no. 3, pp. 1581-1590, Aug. 2011.

[19] Q. Wang, J.-P. Watson, and Y. Guan, "Two-stage robust optimization for N-k contingency-constrained unit commitment," IEEE Trans. Power Syst., vol. 28, no. 3, pp. 2366-2375, Aug. 2013.

[20] MISO, MISO Energy and Operating Reserve Markets, Business Practices Manual, BPM-002-r11, Jan. 2012. [Online]. Available: https: //www.misoenergy.org/_layouts/MISO/ECM/Redirect.aspx?ID=19178

[21] ERCOT, ERCOT Protocols Section 7: Congestion Management, Jul. 2010 [Online]. Available: http://www.ercot.com/content/mktrules/protocols/ current/07-070110.doc

[22] F. Wang and K. W. Hedman, "Reserve zone determination based on statistical clustering methods," in North Amer. Power Symp., Sep. 2012

[23] E. Cotilla-Sanchez, P. D. H. Hines, C. Barrows, S. Blumsack, and M. Patel, "Multi-attribute partitioning of power networks based on electrical distance," IEEE Trans. Power Syst., vol. PP, no. 99, 2013.

[24] K. Purchala, E. Haesen, L. Meeus, and R. Belmans, "Zonal network model of European interconnected electricity network," in CIGRE/IEEE PES Int'l Symp., New Orleans, Louisiana, Oct. 2005.

[25] T. Zheng and E. Litvinov, "Contingency-based zonal reserve modeling and pricing in a co-optimized energy and reserve market," IEEE Trans. Power Syst., vol. 23, no. 2, pp. 277-286, May 2008.

[26] J. D. Lyon, K. W. Hedman, and M. Zhang, "Reserve requirements to efficiently manage intra-zonal congestion," Aug. 2013, IEEE Trans. Power Syst., accepted for publication.

[27] PJM, PJM Manual 10: Pre-Scheduling Operations v26, Nov. 2013. [Online]. Available: http://www.pjm.com/ /media/documents/manuals/ m10.ashx

[28] WECC, Standard BAL-002-WECC-1 - Contingency Reserves, Oct. 2008 [Online]. Available: http://www.nerc.com/files/BAL-002-WECC-1.pdf

[29] B. Stott, J. Jardim, and O. Alsaç, "DC power flow revisited," IEEE Trans. Power Syst., vol. 24, no. 3, pp. 1290-1300, Aug. 2009.

[30] A. Korad and K. W. Hedman, "Robust corrective transmission switching for system reliability," IEEE Trans. Power Syst., 2013, accepted for publication.

[31] G. M. Geoffrion, "Generalized benders decomposition," Journ. of Opt Theory and Apps., vol. 10, no. 4, pp. 237-260, 1972.

[32] C. Grigg et al., "The IEEE reliability test system - 1996," IEEE Trans. Power Syst., vol. 14, no. 3, pp. 1010-1020, Aug. 1999.

[33] Univ. of Washington, "Power systems test case archive," 1999. [Online] Available: http://www.ee.washington.edu/research

Joshua D. Lyon (S'10) received the B.S.E. degree in Industrial Engineering from Arizona State University in 2009. He is currently a Ph.D. student at ASU majoring in Operations Research. Joshua has interned at ISO-NE working on security analysis. His interests include integrating optimization into decision processes and energy markets.

Muhong Zhang received her B.S. in Applied Mathematics from Beijing University of Chemical Technology, China, in 1999, an M.A. in Operations Research from Chinese Academy of Sciences, in 2002 and a Ph.D. in Industrial Engineering and Operations Research from University of California, at Berkeley, in 2006. Currently, she is an assistant professor in the Department of Industrial Engineering in the Ira A. Fulton School of Engineering at Arizona State University.

Kory W. Hedman (S'05-M'10) received the B.S. degree in electrical engineering and the B.S. degree in economics from the University of Washington, Seattle, in 2004 and the M.S. degree in economics and the M.S. degree in electrical engineering from Iowa State University, Ames, in 2006 and 2007, respectively. He received the M.S. and Ph.D. degrees in industrial engineering and operations research from the University of California, Berkeley in 2008 and 2010 respectively.

Currently, he is an assistant professor in the School of Electrical, Computer, and Energy Engineering at Arizona State University. He previously worked for the California ISO (CAISO), Folsom, CA, on transmission planning and he has worked with the Federal Energy Regulatory Commission (FERC), Washington, $\mathrm{DC}$, on transmission switching. 